\title{
To Study Vertebral Artery Blood Flow Velocity During Cervical C1-C2 Manual Traction -Mulligan
}

\author{
Shhalini Grover and G.L.Khanna
}

\begin{abstract}
Aim: To investigate the blood flow of vertebral artery at c1-c2 region during manual traction -Mulligan. Method: The focus of the present study is measuring haemodynamic parameters including peak systolic velocity (PSv) and end diastolic velocity (EDv) of vertebral arteries at the sub occipital region of cervical spine in neutral positioning and whether there is change in the hemodynamic parameters during c1-c2 manual traction (mulligan) using Doppler ultrasonography in normal individual mean age $24.68 \pm 2.03$ year. Results: The result showed significance difference $(\mathrm{p}=0.000)$ in blood flow velocity for PSv and EDv between neutral position and during manual traction for left and right vertebral arteries. Conclusion: This technique had no mechanical effect on vertebral artery rather it has an impact on hemodynamic status of artery and are of great value in increasing the knowledge of the possible treatment protocol related to the blood flow changes with the movements of cervical spine.
\end{abstract}

\author{
Shhalini Grover \\ Associate Professor, \\ Department of Physiotherapy, Faculty of Applied Science \\ Manav Rachna International University, Faridabad, India \\ E-mail: shalinigrover.fas@mriu.edu.in \\ G.L.Khanna \\ Professor\& Dean Faculty of Applied Science \\ Manav Rachna International University, Faridabad, India \\ E-mail: dean.fas@mriu.edu.in
}

\author{
Key Words: Hemodynamic, Doppler, \\ EDv, PSv, Spine
}

DOI: $10.18376 /$ jesp/2017/v13/i1/111269

\section{Introduction}

Cervical spine mobilizations and manipulations are commonly used by physiotherapists and other health professionals to treat mechanical neck pain. After manipulation of cervical spine ,minor short term side effects are very usual.( Refshauge 1994 ) Subjects may feel only local discomfort at the cervical spine where mobilization or manipulations are addressed .Other common symptoms like headache, tiredness, and radiating discomfort dizziness or nausea can also be seen in patients(Ernst 2001 and Cagnie2004). Refshauge 1994 hypothesed that there can be ischemia in the area supplied by vertebral artery. The vertebral artery is unique among the neck vessels by virtue of its position with the cervical spine and relationship to the adjacent muscle ,ligaments \& disc etc. It is the major blood supply to musculoskeletal structures of the cervical spine and the cervical spinal cord. (Moore \& Dalley 1999). The vertebral artery is, divided into four parts ; the first part originate from the subclavian artery in the root of the neck to the sixth cervical vertebra, second part courses through the foramina transversaria of the sixth cervical vertebra to the first cervical vertebra; third part is from the foramen transversarium of the first cervical vertebra(atlas) to the dura mater at the foramen magnum also called as suboccipital part and the fourth part lies within the cranium to the pontomedullary border (Abd El-Bary and Dujovny 1995; Anderson \& Bannister 1989) Between the first and second cervical vertebrae, the vertebral artery runs a variably tortuous course. It is at this level that much of the rotation of the cervical spine takes place (Kapandji, 1987; Soames, 1994), 


\section{Journal of Exercise Science \& Physiotherapy, Vol. 13, No. 1, 2017 \\ ISSN: 0973-2020 (Print) I2OR Impact Factor $=5.23$ UGC Approved [no.20489] ISSN: 2454-6089 \\ (Online)}

where the vertebral artery is more prone to compress and stretch without being damaged but excess of compression or stretch can also cause damage of the artery. (Haynes and Milne, 2001; Thiel 1991). However, it is now generally agreed that the natural tortuosity of the artery between the first two vertebrae allows the vessel to stretch without damage during normal rotation of the cervical spine .Also according to Rivett (2004), vertebral arteries are important entity during manual therapy because of its vulnerability of getting stressed in its torturous course in the cervical spine. The suboccipital part of the vertebral artery is susceptible for injury more than the proximal parts of the artery. (Braun et al, 1983 \& Frumkin, 1990). At this suboccipital region of the cervical spine, the artery takes a loop of approximately 90 degree and passes postero-medially from the foramen transversarium to posterior to the lateral mass of the atlas vertebra and passes along the superior surface of the posterior arch of the vertebra before entering the spinal canal. It is almost fixed in position by connective tissue at this region (Mann 2001 \& Mitchell 2003). It is possible that the artery can be stretched across the rim of the foramen transversarium on the contralateral side to cervical rotation (Haynes and Milne, 2001) and can be compressed against the rim of the lateral mass on the same side of cervical rotation(Bolton, Stick, and Lord, 1989 ). There is reduction of blood flow in the vertebral artery in both the case whether it is ipsilateral or contralateral side of cervical movements (Mitchell 2003). Moreover the surrounding connective tissue which otherwise supports the artery may exacerbate this distortion by restraining the artery movement against cervical spine rotation. Thus, the vertebral artery is expected to be mortified in this region. Although the physiological parameters of blood flow in the vertebrobasilar system are well understood, there are few studies in the current literature which emphasis on the sub- occipital part of the artery .Mitchell (2004) The clinical importance and relevance of vertebral artery (VA) blood flow differences associated with cervical spine movements with either rotation, extension or combination of movements (extension and rotation) of cervical spine used by physiotherapists during the treatment of neck disorder are now a days is main focus of research. Many studies have been conducted on vertebral artery blood flow changes in relation to cervical spine rotation ,few studies report decrease of blood flow in vertebral artery in contralateral to the cervical rotation $\mathrm{Li}$ et al (2008) some report shows no changes in blood flow irrespective to the rotation of cervical spine and one of the study shows an increased flow velocity in the vertebral artery during ipsilateral rotation Lich et al (1999) . Arnold 2004 reported that there is decrease of blood flow whether in contralatral or ipsilateral rotation of cervical spine or in pre-manipulative hold (sustatined extension with rotated cervical spine), all these position put greatest mechanical stress on artery and causes significant decrease in peak systolic and end diastolic velocity of blood flow of vertebral artery. Mitchell (2008) found the blood flow measurement is an important factor and very much dependent upon the part of vertebral artery to be insonated. Many studies have choosen suboccipital area to be researched for vertebral artery blood flow measures because this region is considered to be risky for injury during cervical spine rotation and also lead to blood flow compromise to the hind brain thus causing vertebrobasilar insufficiency or ischaemia. For the diagnosis of the hemo-dynamic status in vertebraobasillary artery system, techniques like magnetic resonance angiography, digital subtraction angiography, invasive electromagnetic flow meter (EMF), and color Doppler Ultrasound (CDU) are used. Currently Colour Doppler ultrasonography is the first choice in the examination of the extracranial part of vertebral artery (Ozdemir, et al 2005) .Research has suggested that the Duplex Doppler ultrasound is a safe, non-invasive, costeffective, painless, widely available, and easily administered diagnostic test. It provides an accurate measure of blood flow in real time thereby allowing for the flow of blood to be measured at various points within an artery ( Johnson et al 2007 ). Vertebral artery is relatively non-tapered and has no appreciable branches in the cervical segment, blood flow measurements of vertebral artery are 


\section{Journal of Exercise Science \& Physiotherapy, Vol. 13, No. 1, 2017 \\ ISSN: 0973-2020 (Print) I2OR Impact Factor $=5.23$ UGC Approved [no.20489] ISSN: 2454-6089 \\ (Online)}

accepted as reliable. Flow volume is an important parameter for evaluation of vertebral artery morphological characteristics and posterior circulation perfusion (Kizilkilic et al 2004). Although blood flow volume is the main parameter affecting brain perfusion, relative changes in velocity may be used to reflect changes in volume. Because the internal diameter (and mean cross-sectional area) does not change during normal laminar blood flow but blood flow velocity $(\mathrm{cm} / \mathrm{s}$, i.e., speed of blood flow) and blood flow volume ( $\mathrm{cm} 3 / \mathrm{s}$ i.e. taking the speed of flow with respect to the crossectional area) will vary proportionally. Thus, a measure of changes in blood flow velocity, associated with movements of the cervical spine, can be considered a good indicator of related changes in blood flow volume. (Mitchell 2003). Mean flow velocity and the "modified Pourcelot Index" (ratio between end-diastolic and maximum blood flow velocity) are considered as signs of increased peripheral resistance. Narrowing of the vessel radius distal of the point of insonation reduces diastolic flow before the systolic flow and thereby reduces markedly the modified PI. The normal range for the modified Pourcelot Index (PI) in the VA is 0.12-0.41, as reported by Biedert et a1 1986. A modified PI of less than 0.1 and a difference in blood flow velocity of more than $50 \%$ between the left and right sides were used as diagnostic criteria for intracranial stenosis or hypoplasia of the VA (Karnik et al 1987). Various research studies have been conducted for measuring the changes in blood flow velocity associated with cervical spine rotation in normal and symptomatic subjects at all segments of vertebral artery. This present study is focused on measuring haemodynamic parameters including peak systolic velocity (PSv) and end diastolic velocity (EDv) of vertebral arteries at the suboccipital region of cervical spine in neutral positioning and whether there is change in the haemodynamic parameters during c1-c2 manual traction (mulligan) using doppler Ultrasonography. It may be considered that manual techniques performed closer to the overall neutral position of the cervical spine will have less effect on the arteries. The c1-c2 manual traction is simple mobilization with movements explained by Mulligan 2003 which bring about resolution of vertigo or other signs that are produced with neck movement. The aim of this study is to investigate whether this c1-c2 mulligan traction can bring about the change in the vertebral artery flow in normal asymptomatic subjects measured with ultrasonography. There is no such study investigated blood flow during specific manual therapeutic technique using ultrasonography. So the question arise was to investigate the effect $\mathrm{c} 1-\mathrm{c} 2$ manual traction on blood flow in vertebral arteries in healthy young participants and to find out whether there was any differences in vertebral artery blood flow while giving cervical manual traction (mulligan) at c1-c2 region. This study is focused on normal young adults aged 20 to 30 years.

\section{Material and Methods}

The 57 healthy young adults with mean age $24.68 \pm 2.03$ participated in this study. All the subjects were explained about the aim and objectives of the research. Out of these, seven reported a history of intermittent cervical pain but were not currently experiencing any symptoms of pain, headache or dizziness/ vertigo, five having stiffness/tightness in neck movements while performing the test ,three didn't report for measurement and reading of two subjects could not be taken because of technical issue. So rest 40 were subjected to do the procedure. Any history of serious cervical spine trauma, such as previous fractures, any congenital disorder recognized which could be associated with hypomobility or instability of the upper cervical spine, diagnosed vertebrobasilar artery insufficiency (VBI) were excluded..(Lucy et al 2013.) The included participants had no neck pain or limitation to neck movement. In this study, Color-coded duplex Doppler Ultrasound imaging is used to measure blood flow velocity i.e. peak systolic velocity (PSv) and end diastolic velocity (EDv) of vertebral arteries (left \& right) in centimeters per second $(\mathrm{cm} / \mathrm{s})$. These measurements quantify maximum (PSv) and minimum flow velocity (EDv). All measurements were taken in a echo laboratory using the duplex Doppler US machine (Philips HDI 5000; Philips Medical 
Systems) having a $7.5 \mathrm{MHz}$ linear array transducer. The measurements of vertebral artery (VA) blood flow velocity were performed at the highest insonable part of the vertebral artery near lateral part of c1-c2 cervical region. The subject was positioned in a supine lying with neck placed in neutral position without pillow. The hand-held probe of $7.5 \mathrm{MHz}$ was placed on lateral aspect of right side of neck and moved up till the mastoid process anterior to the sternocleidomastoid muscle.The probe was angled approximately 6o degree and maneuvered to insonate the underlying vertebral artery, so that an audible and the sharpest visual representations of blood flow in the artery could be obtained. This procedure was repeated to insonate the left side vertebral artery. Measurements of blood flow about peak systolic velocity and end diastolic velocity of the left and the right vertebral arteries were recorded. Following this, each subject was given manual traction at c1-c2 (Mulligan mobilization) Mulligan (1993). The measurement for the blood flow (peak systolic and end diastolic velocity) of right and left vertebral arteries were taken again while maintaining the traction force and readjusting the probe as above. This was necessary because it was not possible to measure blood flow continuously during traction. In this way, the best sonograph was recorded for the right and left vertebral artery.

\section{Results}

Descriptive statistics were expressed included mean, standard deviation, standard error and 95\% confidence intervals between baseline and during mobilization position left and right for PSv and EDv. The dependent variables were tested using kolmogorov-Smirnov,s test. As the data were bind to follow the normal distribution, we used the parametric test for analysis. A multiple linear regression model was used to compare the pre and post PSv and EDv. These pair-wise difference were analysed for statistical significance with using paired t-tests. All statistical tests were completed using SPSS 21 version. The significance level was set at 0.000 and following a significant effect, paired t test was carried. The result showed significance difference in blood flow velocity for PSv and EDv between neutral position and during manual traction for left and right vertebral arteries.

Table 1. Mean \pm SD of Pre \& Post (during traction) haemodynamic parameters of right and left vertebral artery

\begin{tabular}{lccccc}
\hline Parameters & N & Pre-Mean & Post mean & t value & P value \\
& & & & & \\
\hline PSv (Rt)cm/sec & 40 & $56.51 \pm 8.45$ & $64.85 \pm 10.87$ & 4.97 & 0.000 \\
PSv $(\mathbf{L t}) \mathbf{c m} / \mathbf{s e c}$ & 40 & $63.335 \pm 10.34$ & $67.83 \pm 11.66$ & 5.72 & 0.000 \\
& & & & & \\
EDv (Rt) cm/sec & 40 & $24.333 \pm 5.37$ & $30.46 \pm 7.77$ & 7.26 & 0.000 \\
& & & & & \\
EDv (Lt) cm/sec & 40 & $25.99 \pm 4.59$ & $29.70 \pm 6.14$ & 6.67 & 0.000 \\
& & & & & \\
\hline
\end{tabular}

PSv- peak systolic velocity; EDv- end diastolic velocity 


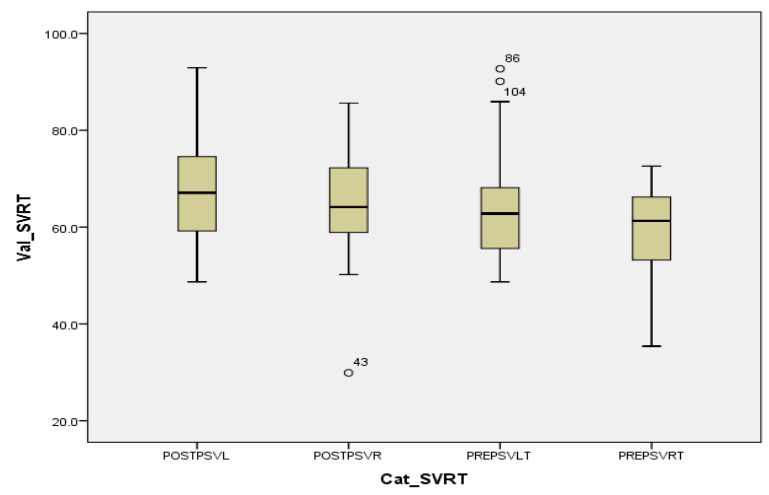

Figure 1. Peak systolic velocity $(\mathrm{rt} \& \mathrm{lt}, \mathrm{cm} / \mathrm{sec})$ of vertebral artery pre and post ( after manual traction)

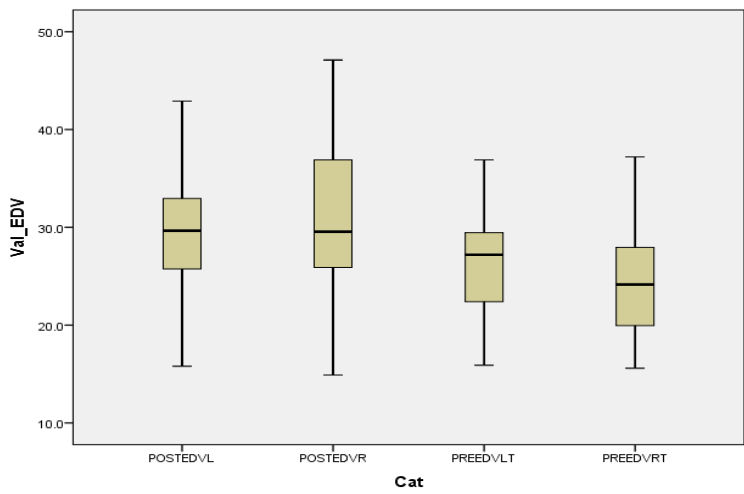

Figur 2. End diastolic velocity $(\mathrm{rt} \& \mathrm{lt}, \mathrm{cm} / \mathrm{sec})$ of vertebral artery pre (neutral) and post( after manual traction)

\section{Discussion}

The present study was focused on measuring hemodynamic parameters including peak systolic velocity (PSv) and end diastolic velocity (EDv) of vertebral arteries at the sub-occipital region of cervical spine in neutral positioning and whether there is a change in the haemodynamic parameters during c1-c2 manual traction (mulligan) using doppler ultrasonography. The findings from this study suggest that the c1-c2 manual traction significantly changes the vertebral artery blood flow velocity in terms of peak systolic velocity (PSv) and end diastolic velocity (EDv). To date, there have been no previous studies investigating blood flow in the vertebral arteries during manual traction using doppler ultrasonography method. Previous studies examining blood flow changes during neck rotation in the vertebral arteries and showed reduction in flow velocity with contralateral rotation Mitchell (2008). An ultrasound study demonstrated marked reduction of vertebral artery flow on neck rotation in some individuals, yet no signs of insufficiency being evoked saying that there is no compromise in cerebral blood supply as the blood supply to the brain is maintained Rivett et al (1999). The study investigated that changes $(<10 \%)$ in blood flow volume normally occur in the anxious state of an individual or while doing a mental task which is not unfavorable to brain functions Kreiger et al (2012). The results of the present experimental 


\section{Journal of Exercise Science \& Physiotherapy, Vol. 13, No. 1, 2017 \\ ISSN: 0973-2020 (Print) $\quad \mathrm{I}_{2}$ OR Impact Factor $=5.23$ UGC Approved [no.20489] ISSN: 2454-6089 \\ (Online)}

study have proved that during intervention of manual traction at c1-c2 level there are statistically significant changes in blood flow velocity. Post intervention, the peak systolic velocity (PSv) of right side vertebral artery increased by $14.7 \%$ and End diastolic velocity (EDv) by $25.19 \%$ and peak systolic velocity (PSv) of left side increased by $7.1 \%$ and End diastolic velocity (EDv) by 14.2\%.Most of the studies have seen the changes in the blood flow with cervical rotation and found the reduction of vertebral artery flow (Haynes, hart and McGeachie 2000; Hynes and Milne 2001). Functional factors such as sustained end range rotation of cervical spine decrease the blood flow in the suboccipital region by distorting the vertebral artery Mitchell J (2005). The present study has investigated the effect of manual traction on blood flow without cervical spine rotation thus to minimize the over stretching and compression of vertebral artery and hence danger to traumatize the vertebral artery was avoided. Peter (1999) demonstrated the effect of cervical manipulation on vertebral artery blood flow in an experimental study done on animals and concluded that there was approximately $20 \%$ increase of blood flow after 20-40 seconds after manipulation of cervical spine. The results of present study shows an increase of blood flow while giving cervical traction for the reason that it has some effect on the length of vertebral artery. The results of the present study were in consistent with the study done by Mangat and Mcdowall (1973) which shows that there is an increase of blood flow of vertebral artery after surgical fusion and post operatively neck traction.

\section{Conclusion}

The manual traction at c1-c2 (Mulligan mobilization) technique was used on asymptomatic sample subjects but in our opinion the symptomatic subjects are the representative of the patient's population on which this technique might be used. None of the subject reported symptoms like vertigo, dizziness, nausea or nystagmus associated with any change in blood flow of vertebral artery. Analysis of the paired comparisons showed that this technique had no mechanical effect on vertebral artery rather it has an impact on hemodynamic status of artery. As such, this study is of great value in increasing the knowledge of the possible treatment protocol related to the blood flow changes with the movements of cervical spine.

\section{Acknowledgment}

I thank Dr. Simmi Manocha Cardiologist Asian Institute of Medical Science and Research for valuable help in doing the ultrasonographic measurement of vertebral artery flow in normal asymptomatic young patients.

\section{Reference}

Arnold C, Bourassa R, Langer T, Stoneham G. 2004. Doppler studies evaluating the effect of a physical therapy screening protocol on vertebral artery blood flow. Manual therapy, Feb.29;9(1):13-21.

Bolton PS, Stick PE, Lord RS.1989. Failure of clinical tests to predict cerebral ischemia before neck manipulation. Journal of Manipulative and Physiological Therapeutics, Aug;12(4):304.

Cagnie B, Vinck E, Beernaert A, Cambier D. 2004. How common are side effects of spinal manipulation and can these side effects be predicted? Manual therapy, Aug 31;9(3):151-6.

Dunne JW, Conacher GN, Khangure MA, Harper CG. 1987.Dissecting aneurysms of the vertebral arteries following cervical manipulation: a case report. Journal of Neurology, Neurosurgery \& Psychiatry, Mar 1;50(3):349-53.

Fast A, Zinicola DF, Marin EL. 1987.Vertebral artery damage complicating cervical manipulation. Spine, Nov 1;12(9):840-2.

Ganong WF. 2005.The autonomic nervous system. Review of Medical Physiology, 22nd ed. New York: McGraw Hill Companies Inc.:223-31. 
Haynes MJ, Milne N. 2001.Color duplex sonographic findings in human vertebral arteries during cervical rotation. Journal of Clinical Ultrasound, Jan 1;29(1):14-24.

Johnson EG, Houle S, Perez A, Lucas SS, Papa D. 2007. Relationship between the duplex Doppler ultrasound and a questionnaire screening for positional tolerance of the cervical spine in subjects with suspected vascular pathology: a case series pilot study. Journal of Manual \& Manipulative Therapy, Oct 1;15(4):225-30.

Johnson CP, Scraggs M, How T, Burns J.1995.A necropsy and histomorphometric study of abnormalities in the course of the vertebral artery associated with ossified stylohyoid ligaments. Journal of clinical pathology, Jul 1;48(7):637-40.

Kapandji IA. 1987. The Physiology of the Joints: Annotated Diagrams of the Mechanics of the Human Joints. Vol. 2, Lower Limb.... Churchill Livingstone.

Krueger BR, Okazaki H. 1980.Vertebral-basilar distribution infarction following chiropractic cervical manipulation. In Mayo Clinic Proceedings, May (Vol. 55, No. 5, pp. 322-332).

Kizilkilic O, Hurcan C, Mihmanli I, Oguzkurt L, Yildirim T, Tercan F. 2004. Color Doppler analysis of vertebral arteries. Journal of ultrasound in medicine, Nov 1;23(11):1483-91.

Krieger SAN, Streicher MN, Trampel R, Turner RD. 2012. Cerebral blood volume changes during brain activation. J. Cereb Blood Flow Metab, 32:1618-1631

Kuether TA, Nesbit GM, Clark WM, Barnwell SL. 1997. Rotational vertebral artery occlusion: a mechanism of vertebrobasilar insufficiency. Neurosurgery, Aug $1 ; 41(2): 427-33$.

Licht PB, Christensen HW, Svendsen P, Høilund-Carlsen PF. 1999.Vertebral artery flow and cervical manipulation: an experimental study. Journal of manipulative and physiological therapeutics, Sep 30;22(7):431-5.

Licht PB, Christensen HW, Højgaard P, Høilund-Carlsen PF. 1998.Triplex ultrasound of vertebral artery flow during cervical rotation. Journal of Manipulative and Physiological Therapeutics, Jan;21(1):27-31.

Licht PB, Christensen HW, Høilund-Carlsen PF. 1999. Vertebral artery volume flow in human beings. Journal of manipulative and physiological therapeutics, Aug 31;22(6):363-7.

Mangat KS, McDowall GD. 1973.Vertigo and nystagmus in cervical spondylosis and the role of' anterior cervical decompression'. The Journal of Laryngology \& Otology, Jun 1;87(06):555-63

Mitchell J. 2005. The vertebral artery: a review of anatomical, histopathological and functional factors influencing blood flow to the hindbrain. Physiotherapy theory and practice, Jan 1;21(1):23-36.

Mitchell JA. 2003. Changes in vertebral artery blood flow following normal rotation of the cervical spine. Journal of Manipulative and Physiological Therapeutics, Aug 31;26(6):347-51.

Mitchell J. 2008.Is mechanical deformation of the suboccipital vertebral artery during cervical spine rotation responsible for vertebrobasilar insufficiency? Physiotherapy Research International, Mar 1;13(1):53-66.

Mitchell J, Kramschuster K. 2008. Real-time ultrasound measurements of changes in suboccipital vertebral artery diameter and blood flow velocity associated with cervical spine rotation. Physiotherapy Research International, Dec 1;13(4):24154. 
Mitchell J. 2005 . The vertebral artery: a review of anatomical, histopathological and functional factors influencing blood flow to the hindbrain. Physiotherapy theory and practice, Jan 1;21(1):23-36.

Mitchell J, McKay A. 1995.Comparison of left and right vertebral artery intracranial diameters. The Anatomical Record, Jul 1;242(3):350-4.

Moore KL, Arthur F. Dalley. Clinically Oriented Anatomy.

Parkin PJ, Wallis WE, Wilson JL. 1978.Vertebral artery occlusion following manipulation of the neck. The New Zealand medical journal, Dec 13;88(625):441.

Palastanga N, Field D, Soames R. 2006. Anatomy and human movement: structure and function. Elsevier Health Sciences.

Rivett DA, Sharples KJ, Milburn PD. 1999. Effect of premanipulative tests on vertebral artery and internal carotid artery blood flow: a pilot study. Journal of Manipulative and Physiological Therapeutics, Aug 31;22(6):368-75.

Rivett DA. 2004.The vertebral artery and vertebrobasilar insufficiency. Bouling JD, Jull GA. Greive's Modern Manual Therapy, The Vertebral Column. Third ed. London: Elsevier Churchill Livingstone, 257-73.

Refshauge KM. 1994. Rotation: a valid premanipulative dizziness test? Does it predict safe manipulation? Journal of Manipulative and Physiological Therapeutics, Jan;17(1):15-9.

Rossiti S, Volkmann R. 1995.Changes of blood flow velocity indicating mechanical compression of the vertebral arteries during rotation of the head in the normal human measured with transcranial Doppler sonography. Arquivos de NeuroPsiquiatria, Mar;53(1):26-33.

Schellhas KP, Latchaw RE, Wendling LR, Gold LH. 1980.Vertebrobasilar injuries following cervical manipulation. Jama, Sep 26;244(13):1450-3.

Selecki BR. 1969. The effects of rotation of the atlas on the axis: experimental work. The Medical journal of Australia, May 17;1(20):1012.

Thiel HW. 1991. Gross morphology and pathoanatomy of the vertebral arteries. Journal of manipulative and physiological therapeutics, Feb;14(2):133-41.

Yi-Kai L, Shi-Zhen Z.1999.Changes and implications of blood flow velocity of the vertebral artery during rotation and extension of the head. Journal of Manipulative and Physiological Therapeutics, Feb 28;22(2):91-5. 